\title{
Promotion of peripheral nerve regeneration of a peptide compound hydrogel scaffold
}

This article was published in the following Dove Press journal:

International Journal of Nanomedicine

2I August 2013

Number of times this article has been viewed

Guo-Jun Wei'
Meng Yao'
Yan-Song Wang'
Chang-Wei Zhou'
De-Yu Wan'
Peng-Zhen Lei'
Jian Wen'
Hong-Wei Lei'
Da-Ming Dong'
'Department of Orthopaedics, The
2nd Affiliated Hospital of Harbin
Medical University, Harbin, People's
Republic of China; 'Department
of Rheumatology, The 2nd Affiliated
Hospital of Harbin Medical University,
Harbin, People's Republic of China

Correspondence: Da-Ming Dong

Department of Orthopaedics, The 2nd

Affiliated Hospital of Harbin Medical

University, Harbin, I5000I, People's

Republic of China

Tel +86 45I 86605599

Email damingdong2009@yahoo.com
Background: Peripheral nerve injury is a common trauma, but presents a significant challenge to the clinic. Silk-based materials have recently become an important biomaterial for tissue engineering applications due to silk's biocompatibility and impressive mechanical and degradative properties. In the present study, a silk fibroin peptide (SF16) was designed and used as a component of the hydrogel scaffold for the repair of peripheral nerve injury.

Methods: The SF16 peptide's structure was characterized using spectrophotometry and atomic force microscopy, and the SF16 hydrogel was analyzed using scanning electron microscopy. The effects of the SF16 hydrogel on the viability and growth of live cells was first assessed in vitro, on PC12 cells. The in vivo test model involved the repair of a nerve gap with tubular nerve guides, through which it was possible to identify if the SF16 hydrogel would have the potential to enhance nerve regeneration. In this model physiological saline was set as the negative control, and collagen as the positive control. Walking track analysis and electrophysiological methods were used to evaluate the functional recovery of the nerve at 4 and 8 weeks after surgery.

Results: Analysis of the SF16 peptide's characteristics indicated that it consisted of a welldefined secondary structure and exhibited self-assembly. Results of scanning electron microscopy showed that the peptide based hydrogel may represent a porous scaffold that is viable for repair of peripheral nerve injury. Analysis of cell culture also supported that the hydrogel was an effective matrix to maintain the viability, morphology and proliferation of PC12 cells. Electrophysiology demonstrated that the use of the hydrogel scaffold (SF16 or collagen) resulted in a significant improvement in amplitude recovery in the in vivo model compared to physiological saline. Moreover, nerve cells in the SF16 hydrogel group displayed greater axon density, larger average axon diameter and thicker myelin compared to those of the group that received physiological saline. Conclusion: The SF16 hydrogel scaffold may promote excellent axonal regeneration and functional recovery after peripheral nerve injury, and the SF16 peptide may be a candidate for nerve tissue engineering applications.

Keywords: peripheral nerve injury, silk fibroin, peptide, hydrogel, scaffold

\section{Introduction}

Peripheral nerve injury (PNI) causes morbidity in about $2.8 \%$ of all trauma patients, leaving many with long-term disabilities due to functional loss and causing major socioeconomic costs. ${ }^{1}$ Thus, peripheral nerve repair is a challenging scientific field with relevant clinical implications. Artificial tissue repair scaffolds may provide a physical guide to allow regenerative axon growth that bridges the lesion cavity and restores functional neural connectivity. By integrating different strategies, including the use of various biomaterials and microstructures as well as incorporation of bioactive molecules and living cells, combined or synergistic effects for spinal cord repair 
through regenerative axon growth may be achieved. ${ }^{2,3}$ For the development of artificial nerve grafts, natural biomaterials have been designed as three-dimensional (3D) scaffolds or hydrogels and further engineered to support neuron survival, adhesion, proliferation, and guidance. These biomaterials included collagen, polyglycolic acid, polylactic acid, chitosan, alginate silk, and their composites or derivatives, etc. ${ }^{4-6}$ These materials are advantageous for the engineering of nerve tissue because they are biocompatible, they favor the migration of supporting cells, and they avoid the occurrence of toxic effects. However, their poor mechanical properties, their high swelling behavior, and their relatively fast in vivo biodegradation rate of natural polymers generally limit their applications. ${ }^{7}$ Thus, seeking new biomaterials with more favorable properties and flexible processing methods remains an active area of research.

Recently, synthetic materials, especially peptide designed from silk fibroin (SF) sequences, have several advantages for their use as tissue engineering scaffolds, including: (1) high stability; (2) low immune response; (3) high surface density and orientation for ligand-receptor interaction and cell adhesion; (4) a relatively low molecular weight; and (5) the ability to be used in high concentrations. ${ }^{8,9}$ These peptides have been studied as biomaterials not only useful for specific 3D tissue cell cultures but also for tissue repair and regenerative therapies. A number of these peptides have been used in the context of peripheral nerve repair, including RGD (arginine-glycine-aspartic acid), a peptide found in fibronectin, laminin and other extracellular matrix (ECM) molecules; IKVAV (isoleucine-lysine-valine-alanine-valine) and YIGSR (tyrosine-isoleucine-glycine-serine-arginine) of the laminin $\beta$ chain, RNIAEIIKDI (arginine-asparagineisoleucine-alanine-glutamic acid-isoleucine-isoleucinelysine-aspartic acid-isoleucine) peptides of the laminin $\gamma$ chain and the primary cell binding domains of laminin; as well as similar peptide sequences such as HAV (histidine-alaninevaline), a mimetic of the $\mathrm{N}$-cadherin regulatory protein that is present on both neurons and glial cells. ${ }^{9,10}$ The use of SF as a substrate for supporting the proliferation of PC12 cells with subsequent cell differentiation by neurite outgrowth has been documented. ${ }^{11}$ Other results have also shown that dissociated dorsal root ganglion neurons can extend long neurites along silk fibers from Bombyx mori silkworms and Schwann cells are able to survive, adhere, and migrate along the silk fibers, suggesting that silk fibroin has potential as a scaffold material to support peripheral nerve regeneration. ${ }^{12}$ Thus, peptide hydrogel scaffolds provide a platform that makes them ideal for nanomedical applications. Peptide hydrogel scaffolds not only have all of the advantages of traditional hydrogels but also do not use harmful materials to initiate the solution-gel transformation, therefore the degradation products are natural amino acids which can be metabolized. ${ }^{10,13,14}$ Although many in vitro and in vivo experiments have demonstrated the advantages of using self-assembly peptide nanofibrous scaffolds in neural tissue engineering, lower mechanical strength and shorter degradation rates present as limitations in specific tissue engineering applications.

In the present study, an SF16 peptide was designed as a biomaterial for developing a novel peptide based compound 3D scaffold. The SF16 hydrogel scaffold was characterized by analysis with atomic force microscopy (AFM) and scanning electron microscopy (SEM), and was assessed in terms of its ability to maintain the viability, morphology and proliferation of PC12 rat pheochromocytoma cells in vitro. Moreover, we also evaluated the efficacy of the SF16 hydrogel scaffold to support neuronal regeneration and axonal sprouting following peripheral nerve injury. Utility of the SF16 peptide incorporated into hydrogel may be a promising candidate for producing $3 \mathrm{D}$ scaffolds to enhance nerve regeneration.

\section{Materials and methods Materials and chemicals}

The SF16 peptide SGGAGGAGGAGGAGGS (theoretical mass $=1088.02 \mathrm{Da}$ ) was commercially synthesized and purified by Shanghai Bootech Bioscience and Technology Co, Ltd (Shanghai, People's Republic of China). The N-terminus and C-terminus of the peptides were protected by acetyl and amino groups, respectively. Peptide solutions were prepared at a concentration of $2 \%$ (weight/volume [w/v]) with Milli$\mathrm{Q}$ water $(18 \mathrm{M} \Omega \cdot \mathrm{cm})$, and stored at $4{ }^{\circ} \mathrm{C}$ before use. Type I collagen was commercially purchased from Merck Millipore (Billerica, MA, USA). The rat pheochromocytoma cell line PC12 was purchased from American Type Culture Collection (ATCC; Manassas, VA, USA). Other chemicals used were of the highest purity available.

\section{Characterization of the SFI6 peptide}

Circular dichroism (CD) of peptides was measured on a Model 400 spectrophotometer (Aviv Biomedical, Inc, Lakewood, NJ, USA) at room temperature. Sample concentrations were approximately $2 \%(\mathrm{w} / \mathrm{v})$, and cells with a path length of $1 \mathrm{~mm}$ were used. AFM was performed at room temperature using the tapping mode on a SPI4000 Probe Station and SPA-400 SPM Unit (Seiko Instruments Inc, Chiba, Japan); $5 \mu \mathrm{L}$ aliquots of the peptide hydrogel and 
$5 \mu \mathrm{L}$ of the peptide solution were evenly deposited onto a freshly cleaved mica surface, respectively. Sample was left on the mica surface for about 20 seconds. The surface was then rinsed with $200 \mu \mathrm{L}$ of Milli-Q water to remove unattached peptide. The samples were covered with petri dishes to avoid contamination and then air-dried for AFM observation. The particle-size distribution of fibers in solution was recorded using a LB-550 dynamic light scattering particle-size analyzer (HORIBA, Ltd, Kyoto, Japan).

\section{Hydrogel preparation, cell culturing and proliferation}

For preparing hydrogel, $20 \mathrm{~mL}$ of peptide solution and $2 \mathrm{~mL}$ of diglycidyl ether stock solutions were mixed homogenously, and the mixtures were adjusted to $\mathrm{pH} 6.5$ using $10 \mathrm{mM}$ hydrochloric acid. Constructs for analysis by SEM were fixed in phosphate buffered saline (PBS) containing $2 \%$ glutaraldehyde, dehydrated, snap fractured, and critical point dried for 2 hours using $\mathrm{CO}_{2}$ at $38^{\circ} \mathrm{C}$ and 1200 psi. The sample was mounted, splutter coated with gold and examined under SEM (S-4800, Hitachi, Japan).

A $250 \mu \mathrm{L}$ volume of hydrogel was pipetted into the center of a 24-well cell culture insert (BD Falcon ${ }^{\mathrm{TM}}$, BD Biosciences, San Jose, CA, USA) and incubated for at least 24 hours to allow for gelatinization. Media $(4 \times 400 \mu \mathrm{L})$ was carefully layered onto the hydrogel with a pipette tip by running it slowly down the inner wall of the insert. The hydrogel was left to allow for $\mathrm{pH}$ equilibration prior to cell plating.

PC12 cells were trypsinized, centrifuged, and resuspended in culture medium with a final volume of $200 \mu \mathrm{L}$. The media above the hydrogel was removed, and then the cells were pipetted onto the insert, being careful not to disturb the hydrogel. Media $(900 \mu \mathrm{L})$ was added below each insert. The initial PC12 seeding density for both SF16 peptide and collagen gel was $5 \times 10^{6}$ cells $/ \mathrm{mL}$. Cells were incubated, and the media was exchanged approximately every 2 days. The morphology and proliferation of cells in hydrogel cultured under various media were examined using an inverted phase contrast microscope (Olympus, Tokyo, Japan). Proliferation of PC12 cells in the SF16 peptide and collagen hydrogel groups was evaluated by fluorometric DNA assay. Cell-seeded hydrogels at days 1, 7, and 14 post culture were harvested and assayed for DNA content with Hoechst dye 33258 (Sigma-Aldrich, St Louis, MO, USA). The DNA content of each sample was measured with a spectrofluorometer by mixing digests $(100 \mu \mathrm{L})$ with $2 \mathrm{~mL}$ of Hoechst 33258 dye/buffer solution.

\section{Surgical procedure}

Female Sprague Dawley rats of approximately 250-300 g were used for this study. All animals were divided into three groups. Group $1(\mathrm{n}=16)$ received $20 \mu \mathrm{L}$ of physiological saline into their right sciatic nerve gap and served as a negative control. Group $2(\mathrm{n}=16)$ received $20 \mu \mathrm{L}$ of SF16 peptide hydrogel. Group $3(\mathrm{n}=16)$ received $20 \mu \mathrm{L}$ of collagen and served as a positive control. Animals were deeply anesthetized with $40 \mathrm{mg}$ of sodium pentobarbital per $\mathrm{kg}$ in the surgical procedure. Surgery was conducted on the rat's right leg, under aseptic conditions. After an incision was made to the skin, the sciatic nerve was exposed by making a muscle incision. The nerve was carefully dissected free of surrounding tissue, and a $10 \mathrm{~mm}$ nerve segment was excised with scissors. The defect was repaired with a $12 \mathrm{~mm}$ silicone tube (1.5 mm inner diameter [id]), which contained 8-0 nylon microsutures inserted into the lumen. The ends of the injured nerve were sutured at a distance of $1 \mathrm{~mm}$ into the ends of the tube, leaving a $10 \mathrm{~mm}$ distance between the ends of the nerve. Using a microsyringe, the tube was filled with the previously described hydrogels.

\section{Electrophysiological study}

Electrophysiological evaluation was made at 4 weeks after surgery. Animals were anesthetized and the sciatic nerve was exposed. The sciatic nerve was stimulated with supramaximal stimulus intensity through a pair of needle electrodes placed directly on the sciatic nerve trunk, $10 \mathrm{~mm}$ proximal to the transection site. Amplitudes of the evoked muscle action potentials were recorded from gastrocnemius muscles with needle electrodes, linked to an evoked potential measuring system (MEB-9200, Neuropack, Nihon Kohden Co, Tokyo, Japan). The amplitude value could be considered to reflect the amount of activated fibers.

\section{Evaluation of regenerated myelinated axons}

Nerve sections were observed under a light microscope, and photographs were taken using a digital camera. The cross-sectional area of the whole nerve section was imaged under $400 \times$ magnification. Ten randomly selected fields with an area in each nerve sample were observed, the analyses conducted using an image analysis system (Image-Pro Plus, Media Cybernetics Company, Rockville, MD, USA) to determine the number of regenerated and myelinated axons, the thickness of the myelin sheath and the diameter of axons. Then, axon counts were extrapolated by using the area algorithm to estimate the total number of axons for each nerve. 


\section{Statistical analysis}

All the experimental data were expressed as the mean \pm standard deviation. Statistical analysis was performed with a two-tailed Student's $t$-test using the Statistical Package for the Social Sciences (SPSS version 11.5, SPSS Inc, Chicago, IL, USA).

\section{Results and discussion}

\section{Characteristics of the SFI6 peptide}

A series of peptides designed from SF have been developed as 3D hydrogel scaffolds or hydrogels, and these silk hydrogels have been shown to support cell viability, morphology and growth. The SF fibers were 3 orders of magnitude smaller than most biopolymer microfibers, and therefore they may provide an applausive environment for cells. ${ }^{9}{ }^{90} \mathrm{We}$ designed a novel SF peptide whose sequence was extracted from the highly repetitive sequences of the heavy chain of SF (SGGAGGAGGAGGAGGS, SF16). CD studies performed on the SF16 peptides showed a strong negative peak near $194 \mathrm{~nm}$ and a weak negative peak near $223 \mathrm{~nm}$ (Figure 1). This spectrum indicated the absence of either an $\alpha$-helix or a $\beta$-sheet in the secondary structure of the oligopeptide, in agreement with some previous literature, with this type of CD spectrum representing a random-coil structure. ${ }^{15}$ Chemical structure and space-filling models of the SF16 peptide were formulated when it was dissolved in deionized water (Figure 2). This dissolution gave rise to a milky translucent appearance at a $\mathrm{pH}$ of approximately 3.4. AFM analysis showed that the SF16 peptide forms a long self-assembled nanofiber fragment. Our results showed that these nanorods had diameters of up to $\sim 80 \mathrm{~nm}$ and lengths of up to $\sim 5 \mu \mathrm{m}$ (Figure 3). Dynamic light scattering measurements were

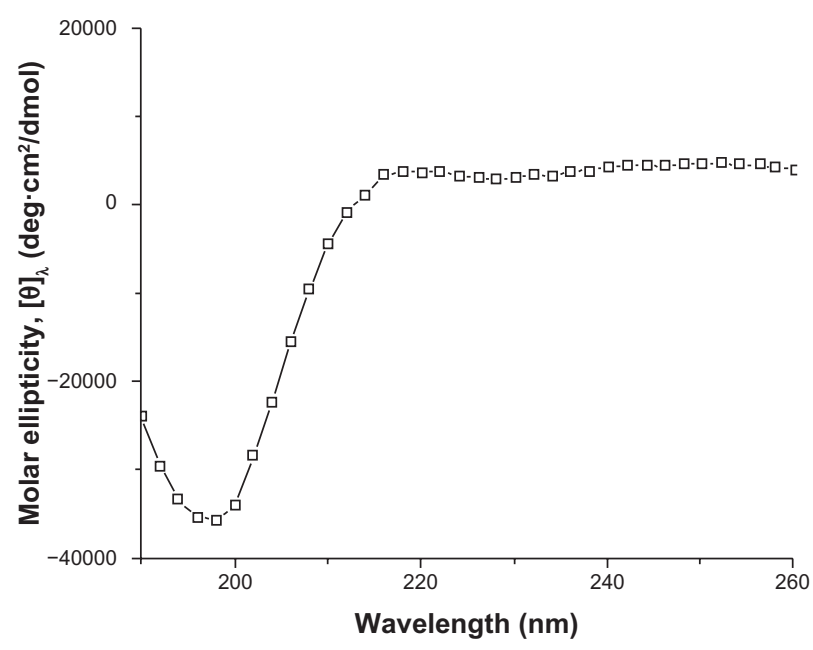

Figure I Circular dichroic (CD) spectra of the silk fibroin peptide (SFI6).

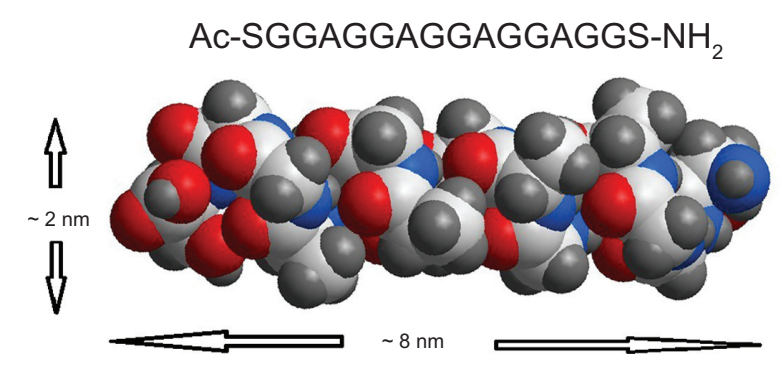

Figure 2 Chemical structure and space-filling model of the silk fibroin peptide (SFI6), with a peptide sequence of SGGAGGAGGAGGAGGS.

carried out to investigate the mechanism by which the SF16 peptide forms a fiber (Figure 4). Our results suggested that the formed bundles of SF16 peptide nanorods had an average length of $\sim 1735 \mathrm{~nm}$, and there was a small fraction distribution $\sim 80 \mathrm{~nm}$ that appeared in the distribution plot of the SF16 peptide. These results strongly indicated the presence of small-sized peptide nanorods/aggregates in selective solvents, and are in agreement with the results of AFM analysis.

\section{Morphology of the SFI6 peptide hydrogel}

For eventual 3D cell culture and in vivo tissue repairing, it is important for hydrogel matrices to have microporous structures so that mass transfer through the matrices is efficient. ${ }^{16} 3 \mathrm{D}$ surface topography and phase AFM images of the SF16 peptide hydrogel are shown in Figure 5. Grains of various sizes that are randomly aggregated can be seen, while owing to the contrast enhancement in phase images, particular grains are clearly highlighted (Figure 5A). From the AFM measurements, phase separation is detected on the surface of the hydrogel and a 3D network structure is formed according to the phase image. As shown in Figure 5B, our results suggested that the SF16 peptide has been successfully integrated into the scaffolds, and exhibited a loose spongelike structure and a cross-linked network. SEM images of the SF16 hydrogel were consistent with the results from the AFM analysis.
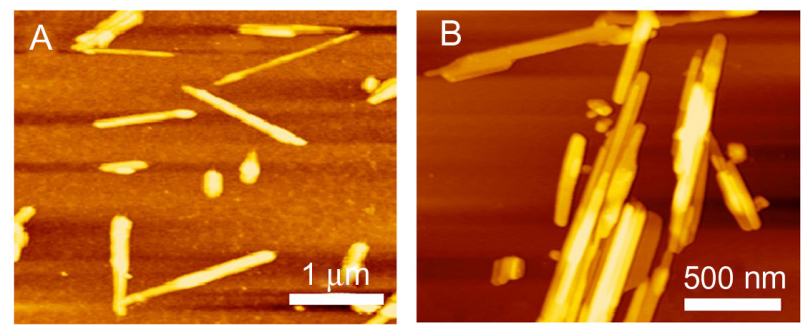

Figure 3 Atomic force microscope images of the silk fibroin peptide (SFI6) nanorods at different scales. (A) $6 \times 6 \mu \mathrm{m}$, (B) $2 \times 2 \mu \mathrm{m}$. 


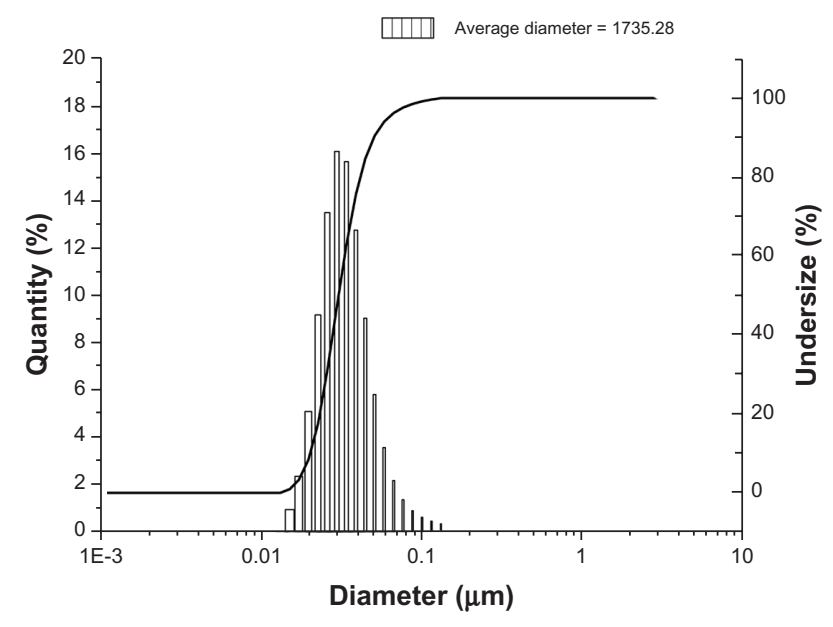

Figure 4 Dynamic laser scattering (DLS) measurements of the silk fibroin peptide (SFI6). DLS measurements of the peptide were carried out at $20^{\circ} \mathrm{C}$. After 2 hours the peptide solution was centrifuged at $12,000 \mathrm{rpm}$ for 15 minutes, and the supernatants produced were used in the measurements.

In the in vivo condition, cells are located in 3D microenvironments, where they are a complex mixture of pores, ridges, and fibers of ECM at nanometer scales. Such structures act as a scaffold to support cells, allow transport of nutrients and metabolic wastes and promote tissue development. ${ }^{16}$

The results suggested that the structure of SF16 peptide hydrogel has similar interior porous morphology consisting of honeycomb-like pores with the pore diameters ranging from 2 to $5 \mu \mathrm{m}$. Our findings indicated that the porous structure of the SF16 peptide hydrogel may show potential as a scaffold for cell infiltration and growth in vitro.

\section{D cell culture}

As shown in Figure 6, cell distribution in the hydrogels is relatively homogenous, and the cell numbers gradually increased with the increased culture times compared to those of day 1 . At day 7, the viable cells had a rounded morphology and clusters were observed in the collagen and SF16 peptide hydrogels. There were no significant differences in the viabilities of cells in the SF16 peptide hydrogel compared to that of the collagen hydrogel. The SF16 peptide scaffold in our
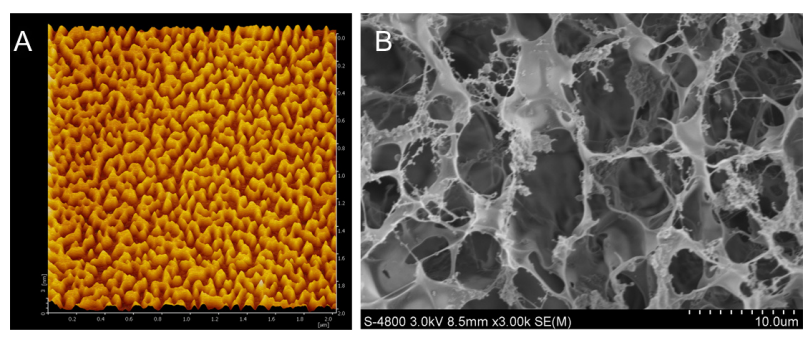

Figure 5 Atomic force microscope (A) and scanning electron microscope (B) images of the silk fibroin peptide (SFI6) hydrogel scaffold.

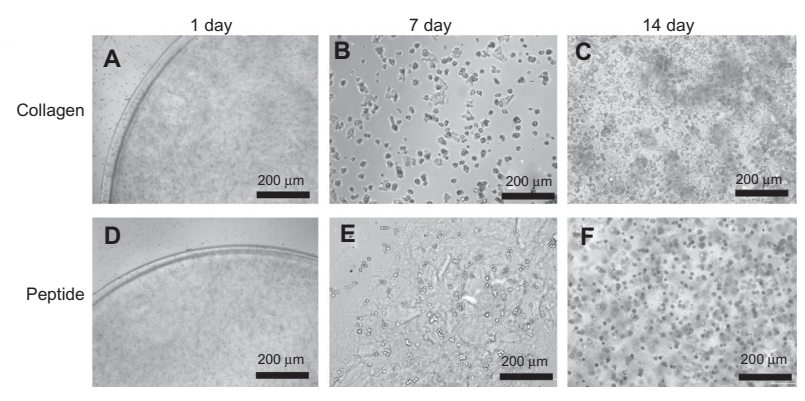

Figure 6 Morphology and viability of chondrocytes cultured in collagen and silk fibroin peptide (SFI6) hydrogel for I, 7 and I4 days. Cells cultured in the hydrogel could be observed to form cell islets and aggregates with a spherical shape.

experiments created a permissive environment for PC12 cell growth. Thus, these results suggested that the SF16 hydrogel may interact with cells to influence cell viability, morphology and proliferation. In addition, we demonstrated that the SF16 peptide hydrogel was not cytotoxic to PC12 cells.

To assess cell proliferation at the culture days 1,7 , and 14, DNA contents were also measured. As shown in Figure 7 , DNA content of the PC12 cells cultured in the SF16 peptide and collagen hydrogels increased gradually with the rising culture time up to day 14; the highest values being about 3.68 and 5.19 times those of the controls, respectively. At day 7 and 14, DNA content in the SF16 peptide hydrogel was lower than in the collagen hydrogel, and the data show no significant differences. Based on the above results, these findings suggested that the SF16 peptide hydrogel has the ability to induce the growth and proliferation of PC12 cells compared to the collagen hydrogel. Earlier results have suggested that a higher degree of PC12 cell proliferation is observed with the other hydrogels. ${ }^{17-20}$ These results contradict the conventional idea that peptide hydrogels might not provide a favorable

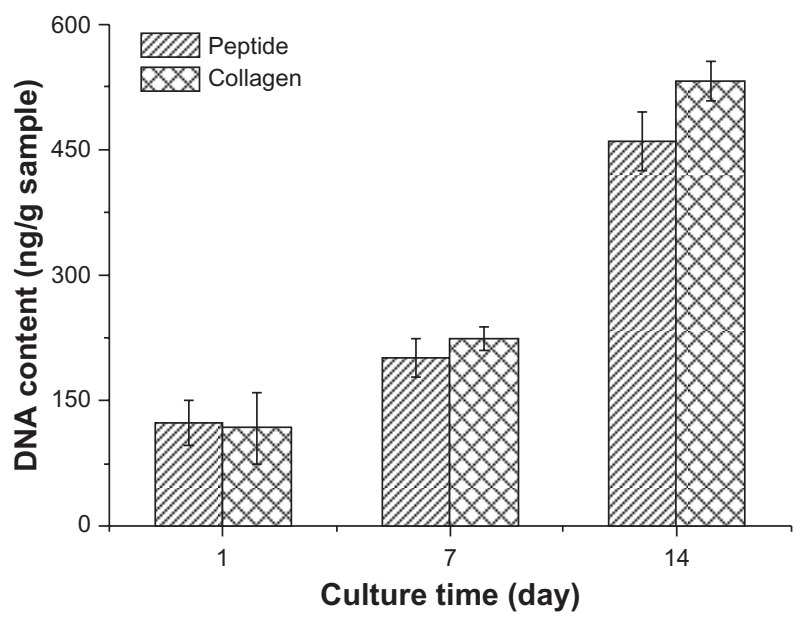

Figure 7 DNA content of $\mathrm{PCI} 2$ cells cultured in the collagen and silk fibroin peptide (SFI6) hydrogel for I, 7 and 14 days, respectively. 


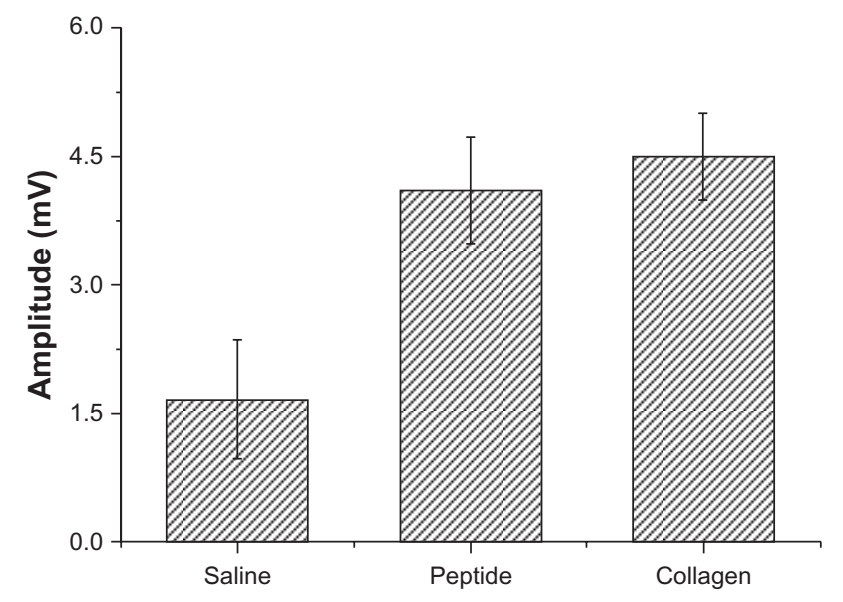

Figure 8 Comparisons of electrophysiological recordings of compound muscle action potential (CMAP) for the silk fibroin peptide (SFI6) and collagen hydrogels at 4 weeks. All results were normalized to the uninjured contralateral reference and reported as mean \pm standard error of the mean.

environment for proliferation of PC12 cells. However, the mechanism by which the SF16 peptide hydrogel environment promoted cell proliferation is complex and requires further study.

\section{Electrophysiological evaluation}

Electrophysiology is a clinical standard to evaluate nerve regeneration. Nerve conduction is based on the bioelectrical action of myelinated axons, and the compound muscle action potential is the response to the number of activated nerve fibers. ${ }^{21}$ Thus, the results of nerve conduction and compound muscle action potential may be considered as evaluation parameters. Our results suggested that the amplitude recovery in the SF16 peptide and collagen hydrogels was significantly higher than that of in the physiological saline group. At 4 weeks, the amplitude recovery values in the saline, SF16 peptide and collagen hydrogels groups were $1.7 \pm 0.8 \mathrm{mV}$,
$4.2 \pm 0.7 \mathrm{mV}$ and $4.5 \pm 0.6 \mathrm{mV}$, respectively (Figure 8). Because the compound muscle action potential (CMAP) amplitude represents the number of innervated muscle fibers, its prompt recovery indicates accelerated reinnervation of muscles by axonal regeneration in the SF16 peptide and collagen hydrogel groups. This recovery of function may be likely due to the provisional matrix provided by the SF16 peptide and collagen hydrogels. In the present study, the SF16 peptide and collagen based scaffold improved electrophysiological outcomes significantly, by 2.5 and 2.7 fold, respectively, compared to that of the physiological saline group. Many of the observed improvements in recovery can be attributed to the ability of the hydrogel to serve as a provisional matrix accelerating nerve regeneration. ${ }^{5,22}$ Early establishment of the matrix most likely led to the increased CMAP and axon density seen at 4 weeks. The early establishment of more neuromuscular contacts in the SF16 peptide and collagen hydrogel groups, as evidenced by the increased amplitude of the CMAP, may have further contributed to the increase in myelinated axon density and average diameter seen at 4 weeks.

\section{Histological observations}

In the peripheral nervous system, the nerve cells in the nerve stumps grew to form myelinated axons after the nerve was injured. Thus, the number, arrangement and density of axons are very important factors to evaluate nerve regeneration. ${ }^{23,24}$

At 4 weeks after surgery the nerve specimens were crosssectioned, stained with toluidine blue and observed in a light microscope (Figure 9). The nerve specimens were also made into ultrathin sections for transmission electron microscopy (TEM) observation (Figure 10). The obtained images were analyzed and counted to evaluate the nerve regeneration. As shown in Figures 10 and 11, the axonal growth in the SF16
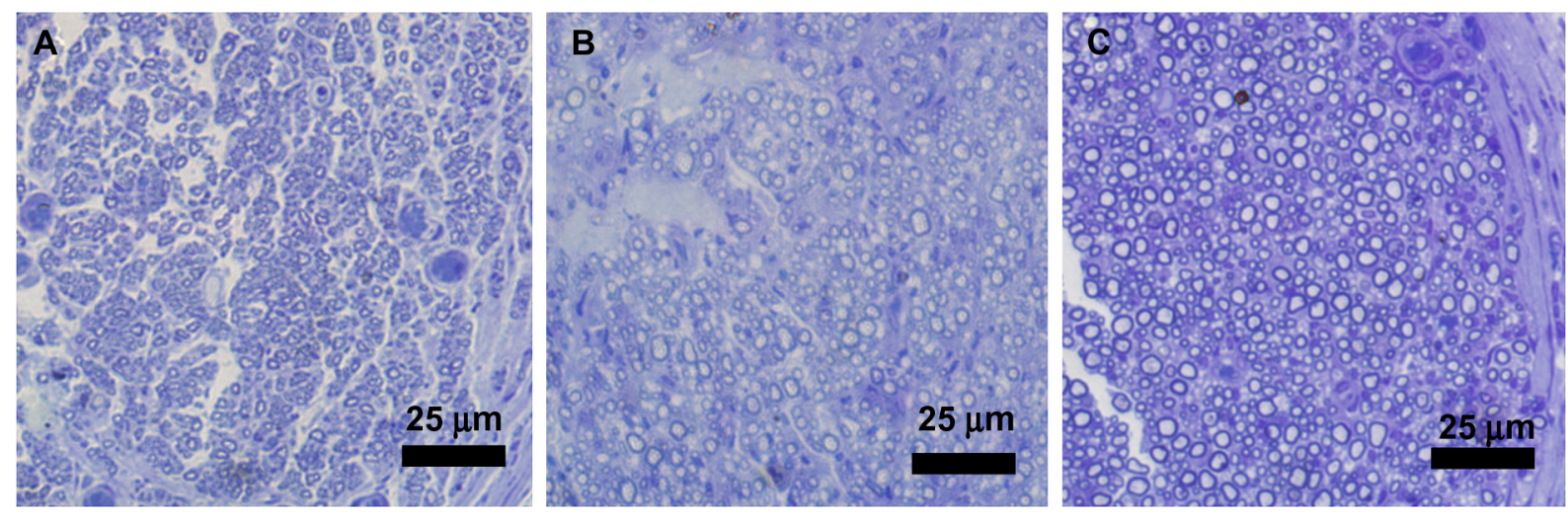

Figure 9 Light micrographs of semi-thin sections showing myelinated axons at the mid-portion of the tubes (4 weeks after surgery, toluidine blue stains). (A) Physiological saline, (B) SFI6 peptide, (C) Collagen. 

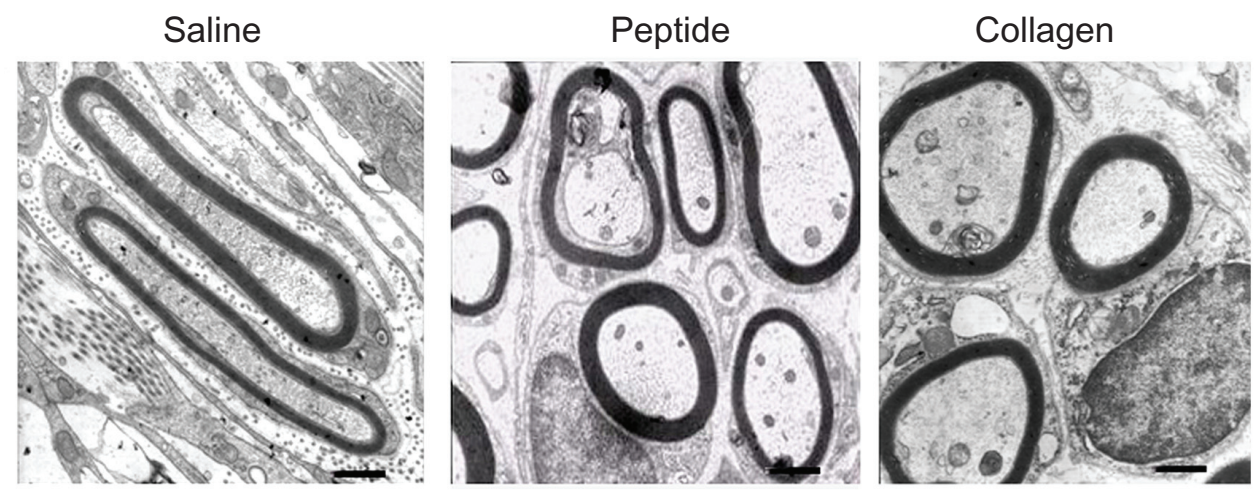

Figure 10 Transmission electron microscopy images of ultrathin sections showing myelinated axons at the mid portion of the tubes at 4 weeks after implantation (bar = I $\mu \mathrm{m}$ ), and comparison of regenerated myelinated axons at 4 weeks after surgery.

peptide and collagen groups showed a faster trend at 4 weeks, a slower trend at 4 weeks, their axonal diameter was larger, their myelin sheath was thicker, and their axonal shape was more regular than those of the physiological saline group. Statistical analysis indicated that there were significant differences in number, diameter, and myelin sheath thickness of nerve fibers between the saline and SF16 peptide groups 4 weeks after surgery, but no significant difference between the SF16 peptide and collagen groups. These results showed that the nerve regeneration in the SF16 peptide and collagen groups was better than in the physiological saline groups. The number of myelinated axons was relatively lower in the physiological saline group at 4 weeks, and these myelinated axons showed poor growth. The number of myelinated axons in the SF16 peptide and collagen groups was increased by $82.9 \%$ and $134.2 \%$ compared to that of the physiological saline group, respectively. For average axon diameter, there was a significant effect of treatment, with the SF16 peptide and collagen hydrogel treated nerves showing significantly increased average myelinated axon diameter. The average diameter in the SF16 peptide and collagen groups represented 1.41 and 1.52 times that of the physiological saline groups. Analysis of myelin thickness showed that the SF16 peptide and collagen hydrogel treated nerves had a larger thickness at 4 weeks than that of the physiological saline group, but there were no significant differences found between the SF16 peptide and collagen hydrogel groups. Thus, the SF16 peptide and collagen hydrogel-treated nerves showed similar nerve regeneration function, and had higher values for the number of myelinated axons, diameter of myelinated axons, and the thickness of myelin sheath compared to those of the physiological saline group. Collagen is characterized by its high mechanical strength, good biocompatibility, and low antigenicity, and it has the potential of being cross-linked with chemicals to make it stronger or to alter its degradation rate. The use of a collagen based matrix has been reported in several studies. ${ }^{4}$ Despite its advantages, collagen may induce immunological and inflammatory responses for some unknown ingredients and/or pathogen from animal origin. As a natural material, its components are difficult to control. However, the designed peptide SF 16 is a chemically synthesized biomaterial, and therefore has the advantage of being a controllable and single ingredient for further applications. ${ }^{8,9}$

Recently, recombinant peptides based on the sequence of SF have shown potential as a scaffold material to support peripheral
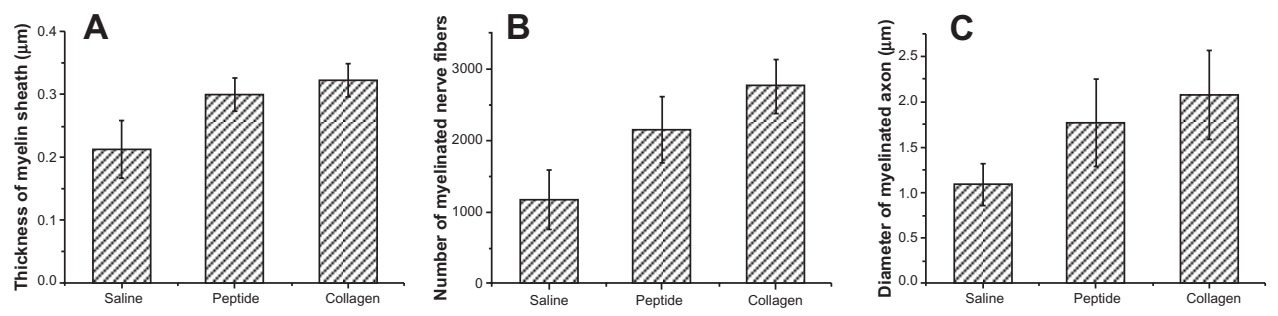

Figure II Comparison of regenerated myelinated axons for the SFI6 peptide at 4 weeks after surgery. (A) The number of myelinated axons ( $n=6$, mean \pm standard error of the mean $[S E M])$. (B) The diameter of myelinated axons based on light micrograph of semi thin sections $(n=6$, mean \pm SEM), and (C) the thickness of the myelin sheath $(n=6$, mean $\pm S E M)$. 
nerve regeneration. ${ }^{25}$ These peptides are completely synthetic, which avoids the potential pathogenicity of animal derived materials. Their degradation products are natural amino acids, which can be metabolized. Moreover, their rapid self-assembly enables cell encapsulation and irregular defect filling in both in vitro and in vivo applications. ${ }^{1-14}$ In the present study, the histological and morphological observations at 4 weeks revealed significant changes in the number, diameter, and myelin sheath thickness of nerve fibers in the SF16 peptide hydrogel group after 4 weeks of implantation, indicating better nerve regeneration. These findings indicate that the SF16 peptide scaffold may have a positive effect in nerve regeneration, and therefore has potential as a matrix for nerve cell propagation.

\section{Conclusion}

The present study suggests that the SF16 peptide consists of a well-defined amino acid sequence that self-assembles under physiological conditions forming a fibrous scaffold within the nanoscale. Its honeycomb like 3D microstructure may be responsible for its ability to control and direct the viability, morphology, and proliferation of PC12 cells in vitro. The SF16 peptide scaffold was found to enhance the amplitude of CMAP, and increase the number and density of axons in tubular nerve sections. Histological analysis suggested that the peptide hydrogel has a positive effect on the number and average diameter of myelinated axons, as well as the thickness of myelin sheath. These positive results indicate that the SF16 peptide might be ideal as a scaffold material for promoting peripheral nerve regeneration. Further research is necessary to improve the design of peptide scaffolds, and could include introducing neurotrophic factors or seeding cells.

\section{Acknowledgments}

This work was supported by grants from Youth Foundation of the 2nd Affiliated Hospital of Harbin Medical University (No QN2010-16).

\section{Disclosure}

The authors declare that they have no conflicts of interest in this work.

\section{References}

1. Raimondo S, Fornaro M, Tos P, Battiston B, Giacobini-Robecchi MG, Geuna $S$. Perspectives in regeneration and tissue engineering of peripheral nerves. Ann Anat. 2011;193:334-340.

2. Biazar E, Khorasani MT, Montazeri N, et al. Types of neural guides and using nanotechnology for peripheral nerve reconstruction. Int $J$ Nanomedicine. 2010;5:839-852.

3. Cunha C, Panseri S, Antonini S. Emerging nanotechnology approaches in tissue engineering for peripheral nerve regeneration. Nanomedicine. 2011;7:50-59.
4. Khaing ZZ, Schmidt CE. Advances in natural biomaterials for nerve tissue repair. Neurosci Lett. 2012;519:103-114.

5. Siemionow M, Bozkurt M, Zor F. Regeneration and repair of peripheral nerves with different biomaterials: review. Microsurgery. 2010;30: 574-588.

6. Biazar E, Khorasani MT, Montazeri N, et al. Types of neural guides and using nanotechnology for peripheral nerve reconstruction. Int $J$ Nanomedicine. 2010;5:839-852.

7. Chiono V, Tonda-Turo C, Ciardelli G. Artificial scaffolds for peripheral nerve reconstruction. Int Rev Neurobiol. 2009;87:173-198.

8. Rymer SJ, Tendler SJ, Bosquillon C, Washington C, Roberts CJ. Self-assembling peptides and their potential applications in biomedicine. Ther Deliv. 2011;2(8):1043-1056.

9. Preda RC, Leisk G, Omenetto F, Kaplan DL. Bioengineered silk proteins to control cell and tissue functions. Methods Mol Biol. 2013;996: $19-41$.

10. Chow D, Nunalee ML, Lim DW, Simnick AJ, Chilkoti A. Peptide-based Biopolymers in Biomedicine and Biotechnology. Mater Sci Eng R Rep. 2008;62:125-155.

11. Uebersax L, Mattotti M, Papaloïzos M, Merkle HP, Gander B, Meinel L. Silk fibroin matrices for the controlled release of nerve growth factor (NGF). Biomaterials. 2007;28:4449-4460.

12. Yang Y, Chen X, Ding F, Zhang P, Liu J, Gu X. Biocompatibility evaluation of silk fibroin with peripheral nerve tissues and cells in vitro. Biomaterials. 2007;28:1643-1652.

13. Krishna OD, Kiick KL. Protein- and peptide-modified synthetic polymeric biomaterials. Biopolymers. 2010;94:32-48.

14. Yan C, Pochan DJ. Rheological properties of peptide-based hydrogels for biomedical and other applications. Chem Soc Rev. 2010;39: 3528-3540.

15. Ranjbar B, Gill P. Circular dichroism techniques: biomolecular and nanostructural analyses - a review. Chem Biol Drug Des. 2009;74: 101-120.

16. Tibbitt MW, Anseth KS. Hydrogels as extracellular matrix mimics for 3D cell culture. Biotechnol Bioeng. 2009;103:655-663.

17. Waddell RL, Marra KG, Collins KL, Leung JT, Doctor JS. Using $\mathrm{PC} 12$ cells to evaluate poly(caprolactone) and collagenous microcarriers for applications in nerve guide fabrication. Biotechnol Prog. 2003;19: 1767-1774.

18. Bender MD, Bennett JM, Waddell RL, Doctor JS, Marra KG. Multi-channeled biodegradable polymer/CultiSpher composite nerve guides. Biomaterials. 2004;25:1269-1278.

19. Park KH, Yun K. Immobilization of Arg-Gly-Asp (RGD) sequence in a thermosensitive hydrogel for cell delivery using pheochromocytoma cells (PC12). J Biosci Bioeng. 2004;97:374-377.

20. Rickett TA, Amoozgar Z, Tuchek CA, Park J, Yeo Y, Shi R. Rapidly photo-cross-linkable chitosan hydrogel for peripheral neurosurgeries. Biomacromolecules. 2011;12:57-65.

21. Navarro X, Udina E. Methods and protocols in peripheral nerve regeneration experimental research: part III-electrophysiological evaluation. Int Rev Neurobiol. 2009;87:105-126.

22. Katayama Y, Montenegro R, Freier T, Midha R, Belkas JS, Shoichet MS. Coil-reinforced hydrogel tubes promote nerve regeneration equivalent to that of nerve autografts. Biomaterials. 2006;27: 505-518.

23. Ellis-Behnke RG, Liang YX, You SW, et al. Nano neuro knitting: peptide nanofiber scaffold for brain repair and axon regeneration with functional return of vision. Proc Natl Acad Sci U S A. 2006;103(13): 5054-5059.

24. Sedaghati T, Yang SY, Mosahebi A, Alavijeh MS, Seifalian AM. Nerve regeneration with aid of nanotechnology and cellular engineering. Biotechnol Appl Biochem. 2011;58:288-300.

25. Venugopal J, Low S, Choon, AT, Ramakrishna S. Interaction of cells and nanofiber scaffolds in tissue engineering. J Biomed Mater Res. 2008;84B:34-48. 
International Journal of Nanomedicine

Dovepress

\section{Publish your work in this journal}

The International Journal of Nanomedicine is an international, peerreviewed journal focusing on the application of nanotechnology in diagnostics, therapeutics, and drug delivery systems throughou the biomedical field. This journal is indexed on PubMed Central, MedLine, CAS, SciSearch ${ }^{\circledR}$, Current Contents ${ }^{\circledR} /$ Clinical Medicine,
Journal Citation Reports/Science Edition, EMBase, Scopus and the Elsevier Bibliographic databases. The manuscript management system is completely online and includes a very quick and fair peer-review system, which is all easy to use. Visit http://www.dovepress.com/ testimonials.php to read real quotes from published authors.

Submit your manuscript here: http://www.dovepress.com/international-journal-of-nanomedicine-journal 